\title{
iTRAQ-based proteomic analysis on the mitochondrial responses in gill tissues of juvenile olive flounder Paralichthys olivaceus exposed to cadmium $^{\text {is }}$
}

\author{
Zhen Lu ${ }^{\text {a, c }}$, Shuang Wang ${ }^{\text {a, c }}$, Chenglong Ji ${ }^{\text {a, b }}$, Fei Li ${ }^{\text {a }}$, Ming Cong ${ }^{\text {a }}$, Xiujuan Shan ${ }^{\text {b }}$, \\ Huifeng $\mathrm{Wu}^{\mathrm{a}, \mathrm{b}, *}$ \\ ${ }^{a}$ CAS Key Laboratory of Coastal Environmental Processes and Ecological Remediation, Yantai Institute of Coastal Zone Research (YIC), Chinese Academy of \\ Sciences (CAS), Shandong Key Laboratory of Coastal Environmental Processes, YICCAS, Yantai, 264003, PR China \\ ${ }^{\mathrm{b}}$ Laboratory for Marine Fisheries Science and Food Production Processes, Qingdao National Laboratory for Marine Science and Technology, Qingdao, \\ 266237, PR China \\ ${ }^{c}$ University of Chinese Academy of Sciences, Beijing, 100049, PR China
}

\section{A R T I C L E I N F O}

Article history:

Received 27 August 2019

Received in revised form

21 October 2019

Accepted 6 November 2019

Available online 9 November 2019

\section{Keywords:}

Cadmium

Paralichthys olivaceus

ITRAQ

Proteomics

Mitochondrial toxicity

\begin{abstract}
A B S T R A C T
Cadmium (Cd) is an important heavy metal pollutant in the Bohai Sea. Mitochondria are recognized as the key target for $\mathrm{Cd}$ toxicity. However, mitochondrial responses to $\mathrm{Cd}$ have not been fully investigated in marine fishes. In this study, the mitochondrial responses were characterized in gills of juvenile flounder Paralichthys olivaceus treated with two environmentally relevant concentrations ( 5 and $50 \mu \mathrm{g} / \mathrm{L}$ ) of Cd for 14 days by determination of mitochondrial membrane potential (MMP), observation of mitochondrial morphology and quantitative proteomic analysis. Both Cd treatments significantly decreased MMPs of mitochondria from flounder gills. Mitochondrial morphologies were altered in Cd-treated flounder samples, indicated by more and smaller mitochondria. iTRAQ-based proteomic analysis indicated that a total of 128 proteins were differentially expressed in both $\mathrm{Cd}$ treatments. These proteins were basically involved in various biological processes in gill mitochondria, including mitochondrial morphology and import, tricarboxylic acid (TCA) cycle, oxidative phosphorylation (OXPHOS), primary bile acid biosynthesis, stress resistance and apoptosis. These results indicated that dynamic regulations of energy homeostasis, cholesterol metabolism, stress resistance, apoptosis, and mitochondrial morphology in gill mitochondria might play significant roles in response to Cd toxicity. Overall, this study provided a global view on mitochondrial toxicity of $\mathrm{Cd}$ in flounder gills using iTRAQ-based proteomics.
\end{abstract}

(c) 2019 Elsevier Ltd. All rights reserved.

\section{Introduction}

Cadmium (Cd) has been confirmed as an important heavy metal pollutants in the marine environments. Due to the development of industry and reckless industrial emissions, Cd has become one of the typical metal pollutants and been posing a risk on economic fishery species in the Bohai Sea (Gao et al., 2014; Li et al., 2018). Although $\mathrm{Cd}$ exhibited its biological function in enzyme synthesis

\footnotetext{
This paper has been recommended for acceptance by Dr. Sarah Harmon.

* Corresponding author. CAS Key Laboratory of Coastal Environmental Processes and Ecological Remediation, Yantai Institute of Coastal Zone Research (YIC), Chinese Academy of Sciences (CAS), Shandong Key Laboratory of Coastal Environmental Processes, YICCAS, Yantai, 264003, PR China.

E-mail address: hfwu@yic.ac.cn (H. Wu).
}

in some marine diatoms (Lane and Morel, 2000), it can induce a variety of toxicities, such as inhibition of growth and reproduction, disruption in energy metabolism and osmoregulation, immunotoxicity and oxidative stress in marine animals, at low levels (ElEbiary et al., 2013; Jin et al., 2015; Xia et al., 2016). Numerous studies have revealed that $\mathrm{Cd}$ treatments could induce significant effects on energy metabolism (Bai and Xu, 2006; Chandurvelan et al., 2017). Especially, our previous work showed that Cd could induce reconstruction of energy homeostasis, stress resistance and apoptosis in marine clam Ruditapes philippinarum, and suggested that mitochondria were the key target of Cd toxicity in clams (Ji et al., 2019).

As it is known, mitochondrion generally is the major energy production center in eukaryotes and involved in many metabolic processes, such as tricarboxylic acid (TCA) cycle, glycometabolism, 
fatty acid metabolism, and apoptosis. Given their complexity, mitochondria are often considered as the toxicity targets of contaminants (Meyer et al., 2013). The mitochondrial toxicity of Cd was reported to induce mitochondrial dysfunction and oxidative phosphorylation (OXPHOS) disturbance by decreasing mitochondrial membrane potential (MMP) and inhibiting ATP synthesis (Dorta et al., 2003; Sokolova et al., 2005a). Many researches confirmed the toxicological effects of $\mathrm{Cd}$ on mitochondria, however, scarcely focused on the comprehensive evaluation of mitochondrial responses to $\mathrm{Cd}$ in marine fishes. Therefore, it is necessary to conduct a global characterization of mitochondrial responses to further understanding the mitochondrial toxicity of $\mathrm{Cd}$ in marine fishes.

As an important economic fishery species in the Bohai Sea, Olive flounder Paralichthys olivaceus has been widely used as experimental species in marine ecotoxicology (Cui et al., 2018; Kim et al., 2004). A few studies have revealed the tissue-specific accumulation of $\mathrm{Cd}$ and its oxidative stress and acute toxicity profile in flounders (Cao et al., 2012; Zhu et al., 2006). However, few studies have focused on the mitochondrial responses to $\mathrm{Cd}$ in flounders at protein level (Ling et al., 2009). Mitochondrial proteomics could provide new insights into mitochondrial function, and has been widely used to investigate the mitochondrial diseases and human ageing (Leahy et al., 2018). Considering the situation of Cd pollution in the Bohai Sea and its effects on fish mitochondria, it is necessary to characterize the global mitochondrial responses to $\mathrm{Cd}$ in flounders at protein level. In this work, the mitochondrial toxicity of Cd was characterized by iTRAQ-based proteomic approach in juvenile flounder $P$. olivaceus after sublethal exposures of $\mathrm{Cd}$ at environmental concentrations ( 5 and $50 \mu \mathrm{g} / \mathrm{L}$ ) for 14 days.

\section{Materials and methods}

\subsection{Experimental animals and exposure}

Juvenile olive flounders were purchased from a fish farm in Yantai, China. After acclimation in aerated normal seawater for one week, 180 fish individuals with similar size (body length $13.49 \pm 0.87 \mathrm{~cm}$, body weight $20.76 \pm 3.82 \mathrm{~g}$ ) were randomly divided into three groups (control and two Cd treatments). The seawater $\mathrm{Cd}$ concentration in some areas in the Bohai Sea was more than $5 \mu \mathrm{g} / \mathrm{L}$ (Gao et al., 2014). For the Cd-treated groups, flounders were therefore exposed to two environmentally relevant concentrations ( 5 and $50 \mu \mathrm{g} / \mathrm{L}$ ) of $\mathrm{Cd}\left(\right.$ in $\mathrm{CdCl}_{2}$ ). Each treatment consisted of two replicate tanks each containing 30 individuals. All tested flounders were cultured in aerated seawater $\left(20 \pm 0.5^{\circ} \mathrm{C}, 32.5 \mathrm{ppt}\right)$ under a $12 \mathrm{~h}$ light and $12 \mathrm{~h}$ dark cycle, fed with pellet feed at $2 \%$ tissue dry weight once a day. Seawater in tanks was replaced by $50 \%$ fresh seawater every $24 \mathrm{~h}$. During the acclimation and exposure periods, no mortality was found in each group. After exposure for 14 days, 60 individual fish from each treatment were anesthetized with ethyl 3-aminobenzoate methanesulfonate (MS-222, 0.15\%), and then gill tissues were immediately dissected for subsequent assays.

\subsection{Cd determination}

The gill samples ( $n=5, \sim 100 \mathrm{mg}$ per sample) of flounders from each group were dried at $80^{\circ} \mathrm{C}$ to constant weights. The dried samples were accurately weighed and then digested in $1 \mathrm{~mL}$ of concentrated nitric acid $\left(\mathrm{HNO}_{3}\right)(70 \%$, Thermo, MA, USA) using a microwave digestion system (Mar5, CEM, NC, USA). Each completely digested sample was diluted with ultrapure water to $10 \mathrm{~mL}$ for Cd determination using inductively coupled plasma-mass spectrometry (ICP-MS) technique (7500i, Agilent, CA, USA). Cd accumulation in gill tissues was defined as $\mu \mathrm{g} / \mathrm{g}$ dry weight $(\mathrm{dw})$. An internal standard (In) was used to correct the sensitivity drift and matrix effects, and a quality control sample $(50 \mathrm{ng} / \mathrm{mL})$ was measured every 10 samples. The recovery of $\mathrm{Cd}$, as tested by three individual spiking experiments, was restricted within $95.5-104.3 \%$ for Cd.

\subsection{Mitochondria isolation}

Gill mitochondria were isolated using the Qproteome ${ }^{\circledR}$ Mitochondria Isolation Kit (Qiagen, Germany) according to the manufacturer's instruction (Fig. S1) with minor modifications. In order to maintain mitochondria alive, all steps were performed at $4{ }^{\circ} \mathrm{C}$. Briefly, the gill tissues were homogenized in ice-cold Lysis Buffer. The homogenate was then centrifuged at $1000 \mathrm{~g}$ for $10 \mathrm{~min}$ to exclude the cytosolic proteins supernatant. The pellet was resuspended in ice-cold Disruption Buffer and disrupted completely using a Dounce homogenizer. The obtained lysate was centrifuged at $1000 \mathrm{~g}$ for $10 \mathrm{~min}$. The pellet containing nuclei, cell debris and unbroken cells was removed. And the supernatant was centrifuged at $6000 \mathrm{~g}$ for $10 \mathrm{~min}$ and the pellet was reserved. The crude mitochondria pellet was finally re-suspended in Mitochondria Storage Buffer for MMP detection. Subsequently, the high-purity mitochondria were separated from the crude mitochondria by density gradient centrifugation. The high-purity mitochondria were finally re-suspended in Mitochondria Storage Buffer for further iTRAQbased proteomic analysis. In order to evaluate the purity of isolated mitochondria, the activities of cytochrome $c$ oxidase (mitochondrial complex IV) in different fractions were assayed with a mitochondrial respiratory chain complex IV activities kit (Genmed, MA, USA). The details were described in the Supporting Materials and Methods S1.

\subsection{Transmission electron microscope observation}

Transmission electron microscope (TEM) analysis was carried out on gill tissues to evaluate the morphological alterations of gill mitochondria induced by $\mathrm{Cd}$. Long longitudinal sections $(\sim 3 \mathrm{~mm})$ from gill filaments near the external area were obtained for observations under TEM (JEM 1200, Japan). Samples were fixed overnight with $2.5 \%$ glutaraldehyde $(\mathrm{v} / \mathrm{v})$ in $0.1 \mathrm{M}$ phosphate buffered solution (PBS, pH 7.4), and kept in 1\% osmium tetroxide for $1 \mathrm{~h}$ for secondary fixation. After being dehydrated in an acetone gradient series, samples were embedded in Epon 812 resins. Ultrathin sections were cut on an Ultracut E ultramicrotome (Reichert-Jung, Austria). Thin sections (70 nm) were mounted on copper grids and stained with aqueous uranyl acetate and lead citrate. Two replicates were performed for each group, and three microscopic fields per replication were then examined under TEM at $80 \mathrm{kV}$. ImageJ software (http://rsb.info.nih.gov/ij/) was used to analyze the mitochondrial density and the mitochondrial area index. The mitochondrial density was the number of mitochondria in a microscopic field and the mitochondrial area index was the total mitochondrial field in a microscopic field.

\subsection{MMP detection}

MMP alternations of crude mitochondria were evaluated with 5,5',6,6'-tetrachloro-1,1',3,3'-tetraethyl benzimidazolyl carbocyanine iodide (JC-1) fluorescent probe (Beyotime, Shanghai, China). JC- 1 is a cationic dye that may exhibit potential-dependent accumulation in mitochondria (Perelman et al., 2012). Normally, JC-1 is a green fluorescent dye in monomer form $(\sim 529 \mathrm{~nm})$ and can form red fluorescent aggregates $(\sim 590 \mathrm{~nm})$ when concentrated in 
energized mitochondria due to their higher membrane potential. Briefly, $0.9 \mathrm{~mL}$ of JC- 1 working solution was fully mixed with $0.1 \mathrm{~mL}$ of crude mitochondria (containing $10-100 \mu \mathrm{g}$ of proteins). Then the MMP was detected by a flow cytometer (Accuri C6 Plus, BD, NJ, USA) at $488 \mathrm{~nm}$ excitation. JC-1 monomers and aggregates were detected in FITC channel $(515-545 \mathrm{~nm})$ and PE channel $(565-605 \mathrm{~nm})$, respectively. Data were processed using BD Accuri C6 Plus software (version 1.0.23.1), and a visual histogram of PE versus FITC was applied to show the MMP in stained mitochondria. The ratio of JC-1 aggregates (P1) to monomers (P2) was calculated $(n=4)$ for each group.

\subsection{Metabolomic analysis}

Polar metabolites in flounder gills ( $n=10$ for each group) were extracted by a modified extraction protocol (Wu and Wang, 2010). Briefly, the gill tissues ( $100 \mathrm{mg}$ wet weight) were homogenized and extracted in $4 \mathrm{~mL} / \mathrm{g}$ of methanol, $5.25 \mathrm{~mL} / \mathrm{g}$ of water and $2 \mathrm{~mL} / \mathrm{g}$ of chloroform. Metabolite extracts of flounder gills were analyzed on an NMR spectrometer (AV III 500, Bruker, Germany) performed at $500.18 \mathrm{MHz}$ (at $25^{\circ} \mathrm{C}$ ) as described previously (Ji et al., 2013). Details of data processing and analysis of metabolomics were described in the Supporting Materials and Methods S2.

\subsection{Mitochondrial proteomic profiling}

The iTRAQ technique was applied to quantitatively investigate proteomic changes induced by $\mathrm{Cd}$ in flounder samples. The highpurity mitochondria from fish gills were dissolved in SDT lysis buffer (4\% SDS, $100 \mathrm{mM}$ Tris-HCl, $1 \mathrm{mM}$ DTT, $\mathrm{pH}$ 7.6) with protease inhibitor. The concentrations of mitochondrial protein extracts were quantified using Bicinchoninic Acid (BCA) Protein Assay Kit (Beyotime, Shanghai, China). After digestion with the filter aided proteome preparation (FASP) method, mitochondrial proteins were labeled using an iTRAQ 8-plex Reagents (AB SCIEX, MA, USA) according to the manufacturer's protocol. Eight samples (two biological replicates for control, and three biological replicates for each Cd treatment) were labeled with the iTRAQ tags for control group (113 tag and $114 \mathrm{tag}$ ), $5 \mu \mathrm{g} / \mathrm{L}$ Cd-treated group (115 tag, 116 tag and $117 \mathrm{tag}$ ), and $50 \mu \mathrm{g} / \mathrm{L}$ Cd-treated group (118 tag, 119 tag and $121 \mathrm{tag}$ ). The iTRAQ-labeled samples were then pooled and fractionated by strong cation exchange (SCX) chromatography. A total of 30 SCX fractions were collected along the gradient and combined into 10 pools. Each pooled fraction was analyzed using the Q-Exactive mass spectrometer coupled with an Easy nLC nanoflow HPLC system (Thermo, CA, USA). The proteins were identified using Proteome Discoverer software (version 1.4, Thermo, CA, USA) with Mascot search engine (version 2.2, MatrixScience, London, UK) searching against a protein database of $P$. olivaceus downloaded from NCBI (established at May 8, 2018). Protein ratios with $p<0.05$ (Student's $t$-test) and a false discovery rate (FDR) $<0.01$ were considered as positively identified proteins. Only proteins with fold changes $>1.2$ or $<0.83$ and $p$ values $<0.05$ were considered as differentially expressed proteins (DEPs). The function of DEPs was evaluated by the database of Gene Ontology (GO) (http://www.geneontology. org/) and the Kyoto Encyclopedia of Genes and Genomes (KEGG) (https://www.kegg.jp/). More details were described in the Supporting Materials and Methods S3.

\subsection{Statistical analysis}

These obtained indices, including $\mathrm{Cd}$ accumulation, mitochondrial densities, mitochondrial area indexes, and MMP alternations in gill samples were expressed as mean \pm standard deviation (SD) and tested for normal distribution (Ryan-Joiner's test) and homogeneity of variances (Bartlett's test). One way analysis of variance (ANOVA) with Tukey's test was conducted on these indices from control and Cd-treated groups, respectively, and a $p$ value less than 0.05 was considered statistically significant. Minitab 15 (Minitab, PA, USA) was used for statistical analysis.

\section{Results and discussion}

\subsection{Cd accumulation in flounder gill tissues}

As the key respiratory organ in fish, gill tissue was regarded as the initial target when encountered aqueous pollutants (Trevisan et al., 2014). As shown in Fig. 1, compared with the control $(0.24 \pm 0.04 \mu \mathrm{g} / \mathrm{g} \mathrm{dw})$, the concentrations of $\mathrm{Cd}$ was significantly $(p<0.01)$ increased in gill samples of Cd-treated groups. Cd contents in both $\mathrm{Cd}$ treatments were $0.82 \pm 0.16 \mu \mathrm{g} / \mathrm{g} \mathrm{dw}$ and $3.81 \pm 1.01 \mu \mathrm{g} / \mathrm{g} \mathrm{dw}$, which were approximately 3 and 15 times higher than that in control group, respectively.

\subsection{Evaluation for mitochondrial purity}

Cytochrome $c$ oxidase (complex IV), a key enzyme in mitochondria, can be used to evaluate mitochondrial purity. In this study, the activity of complex IV was measured in different fractions acquired during the isolation procedure (Fig. S1). As shown in Fig. 2, the activities of complex IV in M1 and M3 were significantly higher than that in $\mathrm{M} 2$, indicating that the gill mitochondria were significantly enriched. The crude and high-purity mitochondria were subsequently used for MMP detection and proteomic analysis, respectively.

\subsection{Effects of $C d$ on $M M P$}

MMP is primarily generated by proton pumps which composed of NADH dehydrogenase (complex I), cytochrome $c$ reductase (complex III) and complex IV. It plays a vital role in generating ATP and maintaining mitochondrial function (Zorova et al., 2018). The ratio of $\mathrm{P} 1 / \mathrm{P} 2$ was used to indicate the MMP of gill mitochondria from different groups (Fig. 3). Compared with the control group $(6.21 \pm 3.64)$, MMPs were significantly reduced in the low concentration $(5 \mu \mathrm{g} / \mathrm{L}, 2.56 \pm 0.79, p<0.05)$ and the high concentration

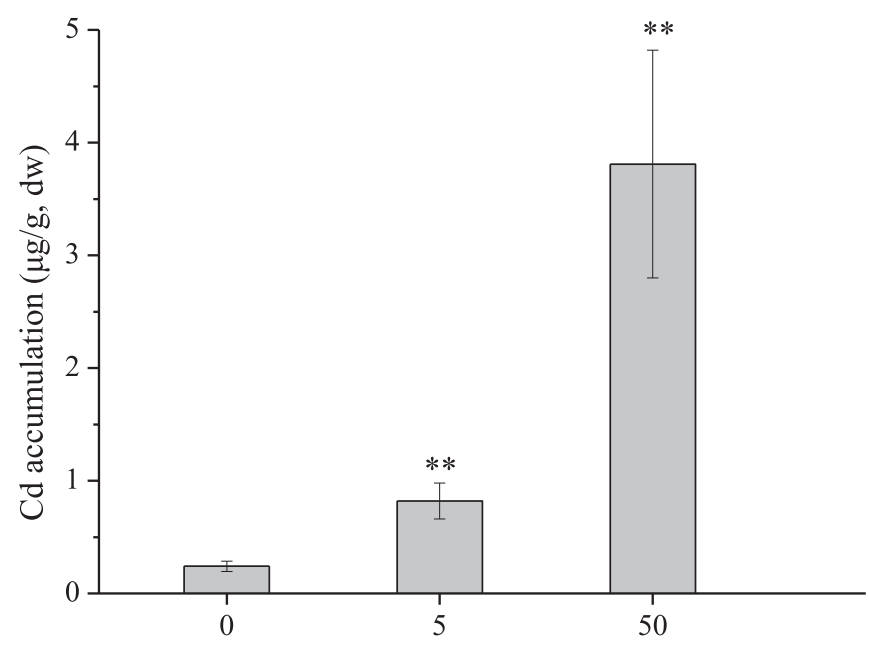

Cd concentration for exposure $(\mu \mathrm{g} / \mathrm{L})$

Fig. 1. The accumulated $\mathrm{Cd}$ concentrations $(\mu \mathrm{g} / \mathrm{g} \mathrm{dw})$ in gill samples $(n=5)$ from control and Cd-treated groups after $\mathrm{Cd}$ treatments for 14 days. Values were presented as mean $\pm \mathrm{SD}$. ${ }^{* *}$ represents $p<0.01$. 


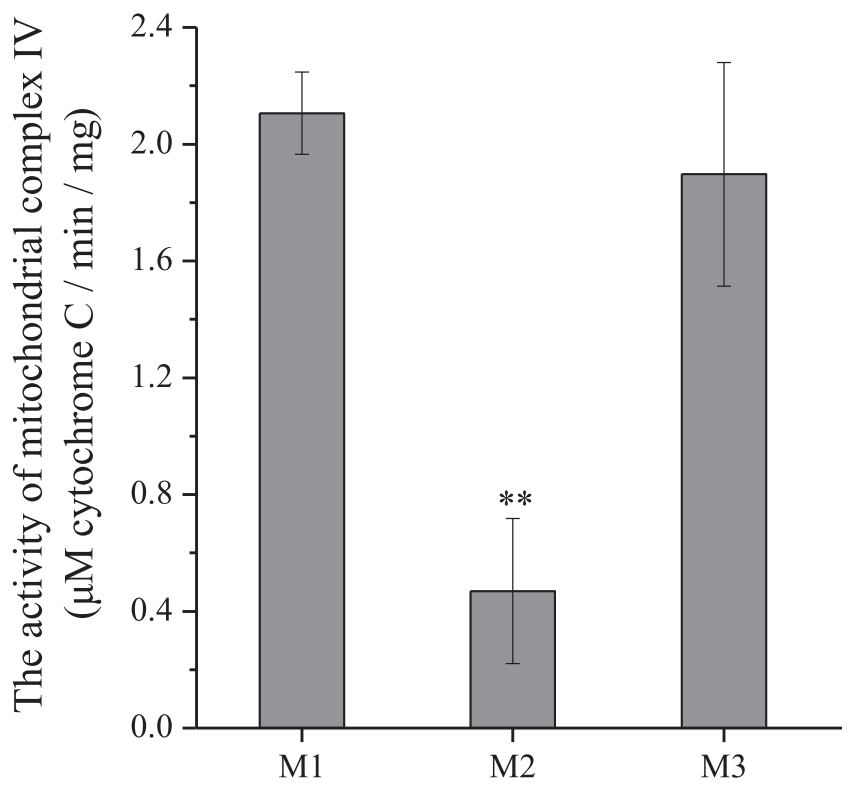

Fig. 2. The activity of mitochondrial complex IV $(n=3)$ in different fractions acquired during the isolation procedure. Values were presented as mean \pm SD. ${ }^{* *}$ represents $p<0.01$. Abbreviations: M1, the whole lysate of gill cells; M2, the discarded pellet which contained nuclei, cell debris and unbroken cells; M3, the reserved suspension of crude mitochondria.

$(50 \mu \mathrm{g} / \mathrm{L}, \quad 1.69 \pm 0.28, \quad p<0.01)$ of Cd-treated groups (Fig. 3). Consistently, decreased MMPs were also observed in eastern oyster Crassostrea virginica and clam Ruditapes philippinarum after $\mathrm{Cd}$ treatments (Ji et al., 2019; Sokolova et al., 2005a). It was reported that $\mathrm{Cd}$ could attach to the mitochondrial inner membrane and enhance its membrane permeability, leading to the efflux of $\mathrm{H}^{+}$ (Sanadi et al., 1981). However, proton gradients are essential for generating ATP (Daichi et al., 2011). Therefore, the mitochondrial membrane depolarization in both $\mathrm{Cd}$ treatments implied that $\mathrm{Cd}$ affected ATP production and induced mitochondrial dysfunction in flounder gills.

\subsection{Overview of mitochondrial proteome alterations induced by $\mathrm{Cd}$ treatment}

To further elucidate the mitochondrial dysfunction induced by Cd treatments, the altered mitochondrial proteins were investigated using iTRAQ-based proteomics. A total of 4690 proteins were identified on the basis of 51,512 matched peptide spectra, of which 19,783 peptides were unique. There were 361 proteins significantly altered in response to $\mathrm{Cd}$ treatments, among which 128 proteins were identified as mitochondrial proteins. The functions of these mitochondrial DEPs were analyzed subsequently to elucidate the mitochondrial toxicity of $\mathrm{Cd}$ in juvenile flounders (Table S1). In details, 74 out of 128 DEPs were detected in the low concentration $(5 \mu \mathrm{g} / \mathrm{L})$ of Cd-treated group, including 72 up-regulated DEPs (fold change $>1.2, \quad p<0.05$ ) and 2 down-regulated DEPs (fold change $<0.83, p<0.05$ ) (Fig. $4 \mathrm{~A}$, Table S1). In the high concentration $(50 \mu \mathrm{g} / \mathrm{L})$ of $\mathrm{Cd}$ treatment, 118 out of 128 DEPs were identified, containing 114 up-regulated and 4 down-regulated DEPs (Fig. 4A, Table S1). There were 64 (50.0\%) common DEPs in both Cd-treated groups (Fig. 4B). Compared with the low concentration $(5 \mu \mathrm{g} / \mathrm{L})$ of Cd-treated group, DEPs with larger numbers and higher fold changes were observed in the high concentration $(50 \mu \mathrm{g} / \mathrm{L})$ of $\mathrm{Cd}$ treated group, indicating the dose-dependent effects of $\mathrm{Cd}$ on gill mitochondria of juvenile flounders.

\subsection{Effects of $\mathrm{Cd}$ on mitochondrial morphology and import}

Mitochondrial morphologies are related to the energy status and cell viability (Karbowski and Youle, 2003). Chlorine cells of teleost gills are reported to be rich in mitochondria (Jurss and Bastrop, 1995). Therefore, chlorine cells were selected for TEM observations in the present study. For the control group, mitochondria showed normal morphological features, with homogeneous shape and size, clear and integrated mitochondrial inner membrane (MIM) and mitochondrial outer membrane (MOM), and densely arranged cristae (Fig. 5A). However, mitochondria of gill tissues in Cd-treated groups presented a series of pathological changes, such as damage or shedding of IM/OM, detachment or irregular arrangement of cristae (Fig. 5B and C). Similarly, mitochondrial damages were also induced by $\mathrm{Cd}$ treatments in freshwater crab Sinopotamon henanense and white sea bass Lates calcarifer (Thophon et al., 2010; Xuan et al., 2014). Besides, compared with the control group, significant dose-dependent increases of mitochondrial density and decreases of mitochondrial area index $(p<0.05)$ were induced by $\mathrm{Cd}$. However, no significant difference was observed between the mitochondrial area indexes from both Cd treatments (Fig. 5D). These findings indicated that $\mathrm{Cd}$ could not only cause mitochondrial ultrastructural damages but also induce more and smaller mitochondria in flounder gills. Similarly, Sokolova et al. (2005b) found that mitochondrial number of eastern oyster Crassostrea virginica increased with the increased concentrations of heavy metals, which was considered as a compensatory mechanism for the respiratory depression caused by heavy metals.

Proteomic analysis revealed that 6 DEPs related to regulating mitochondrial morphology were significantly up-regulated in Cdtreated groups (Table S1). Mitochondrial contact site and cristae organizing system (MICOS) complex play a crucial role in mitochondrial dynamics, cristae maintenance, cristae junctions (CJs) formation and contact sites between MOM and MIM (Harner et al., 2011). MICOS complex subunit mic19 (MIC19), MICOS complex subunit mic60 (MIC60) and MICOS complex subunit mic25 (MIC25) are three main subunits in MICOS complex (An et al., 2012; Darshi et al., 2011; Glytsou et al., 2016). The down-regulation of MIC25 was reported to significantly decrease ATP synthesis in mitochondria (Ding et al., 2015). This protein was commonly up-regulated in both Cd-treated groups, contributing to mitochondrial morphology maintenance and increase of ATP production. MIC60, MIC19 and MICOS complex subunit mic25a-like isoform X2 (MIC25a) were uniquely up-regulated in the low concentration $(5 \mu \mathrm{g} / \mathrm{L})$ of $\mathrm{Cd}$ treated group, which meant more compensation to maintain mitochondrial morphology compared with the high concentration $(50 \mu \mathrm{g} / \mathrm{L})$ of $\mathrm{Cd}$ treatment. Besides, MICOS complex is required for OXPHOS and normal mitochondrial ultrastructure. It was reported that respiratory complexes could assemble and did not function optimally in the absence of the MICOS complex, leading to the respiratory defects possibly caused by decreases of CJs (Friedman et al., 2015). In this work, the up-regulated MICOS subunits might maintain the proper assembly of respiratory complexes. Coiledcoil-helix-coiled-coil-helix domain-containing protein 10 (CHCHD10) is enriched at CJs and critical for maintaining ATP synthesis, oxygen consumption and mitochondrial ultrastructure (Bannwarth et al., 2014; Genin et al., 2016). DnaJ homolog subfamily C member 11 (DNAJC11) was reported to interact with the MICOS complex and involved in the formation of mitochondrial cristae and CJs (Zarouchlioti et al., 2018). These two proteins were up-regulated in flounder samples from the low concentration $(5 \mu \mathrm{g} /$ L) of Cd-treated group, which might contribute to the ATP production and stability of mitochondrial networks.

For mitochondrial import, there were 8 DEPs identified in both 

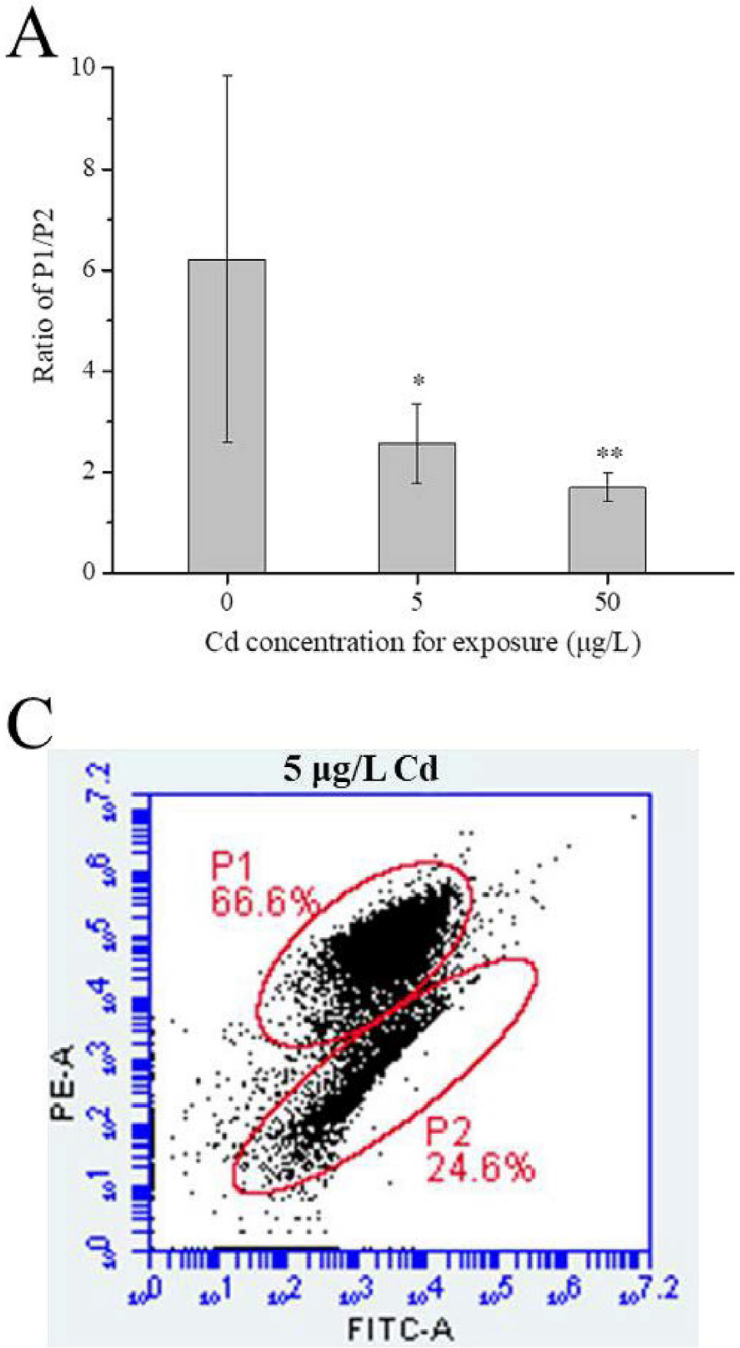

B

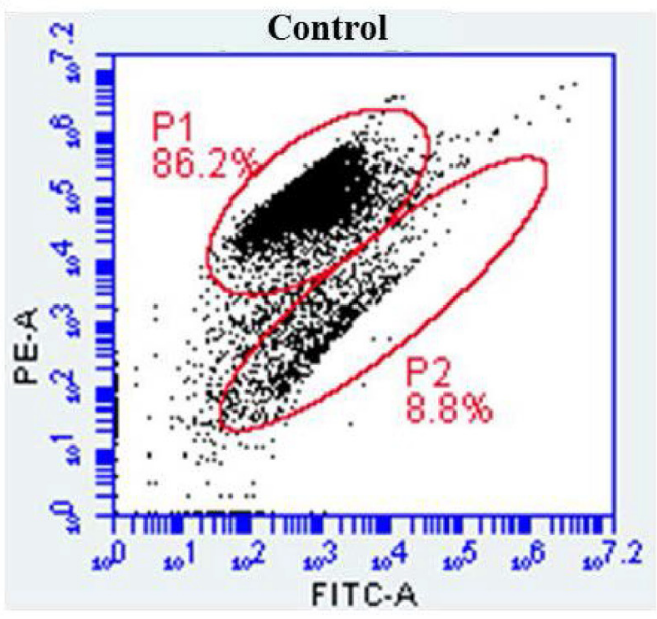

$\mathrm{D}$

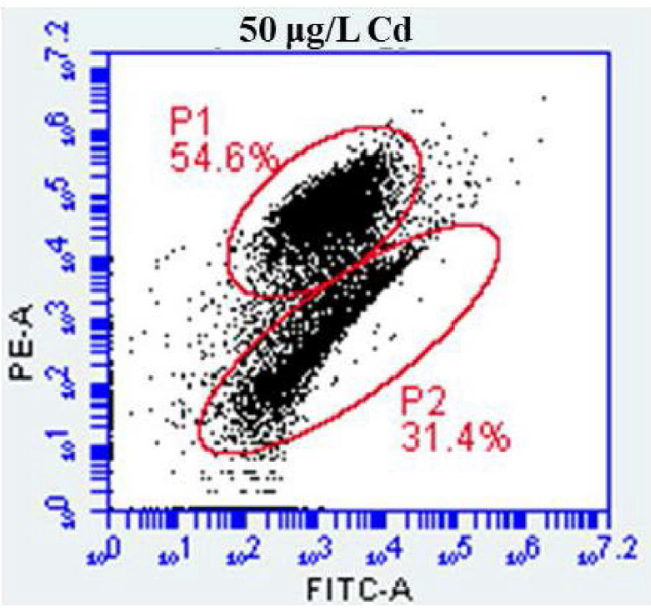

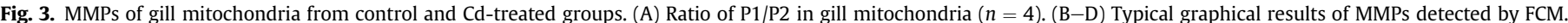
Values are presented as mean \pm SD. ${ }^{*}$ represents $p<0.05,{ }^{* *}$ represents $p<0.01$.

A

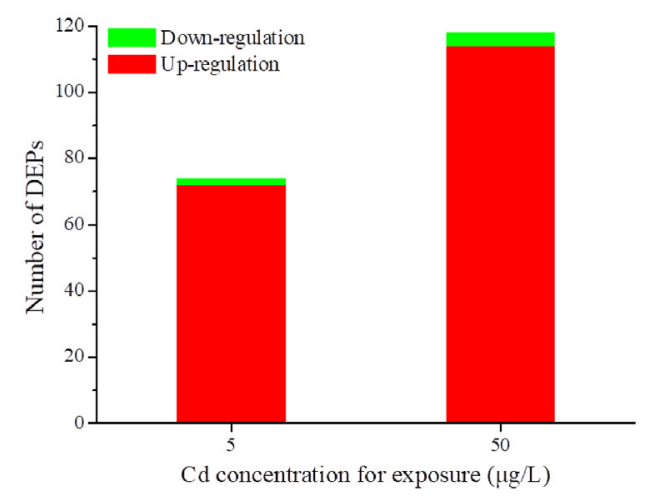

B

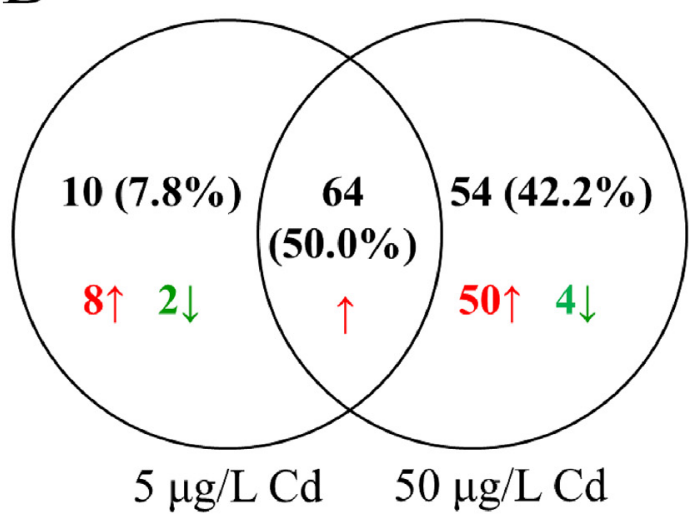

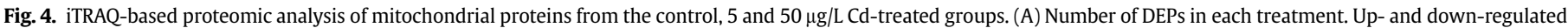

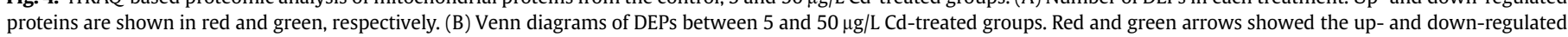
DEPs, respectively. (For interpretation of the references to colour in this figure legend, the reader is referred to the Web version of this article.) 
A

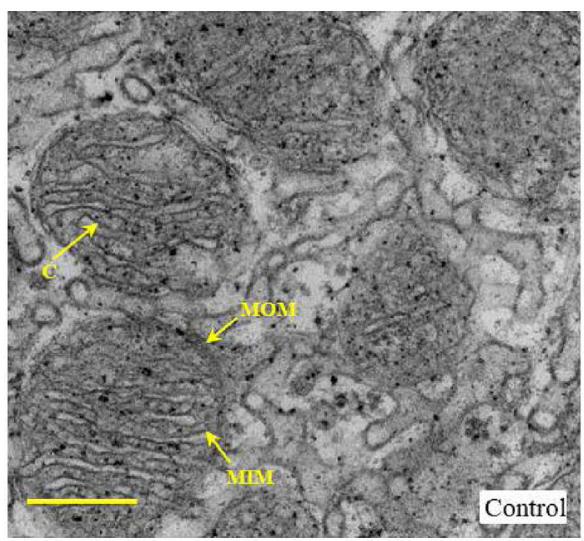

$\mathrm{C}$

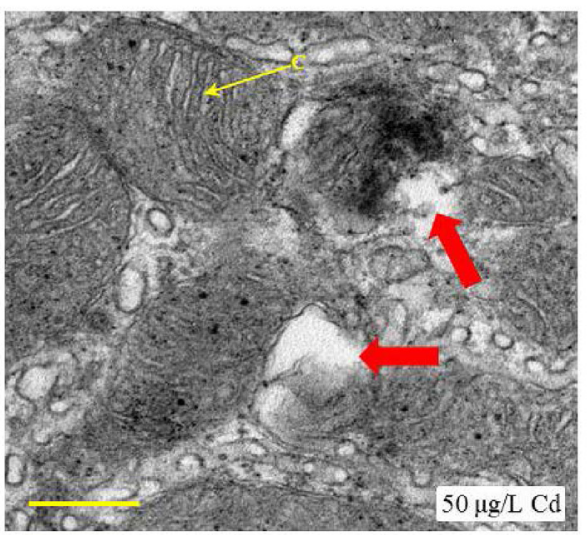

$\mathrm{B}$

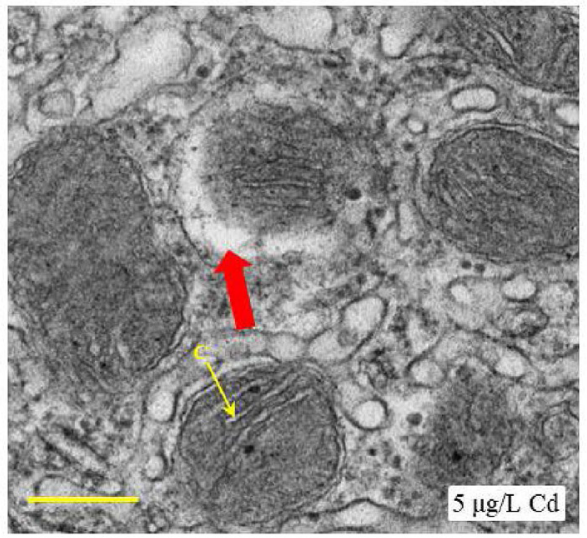

$\mathrm{D}$

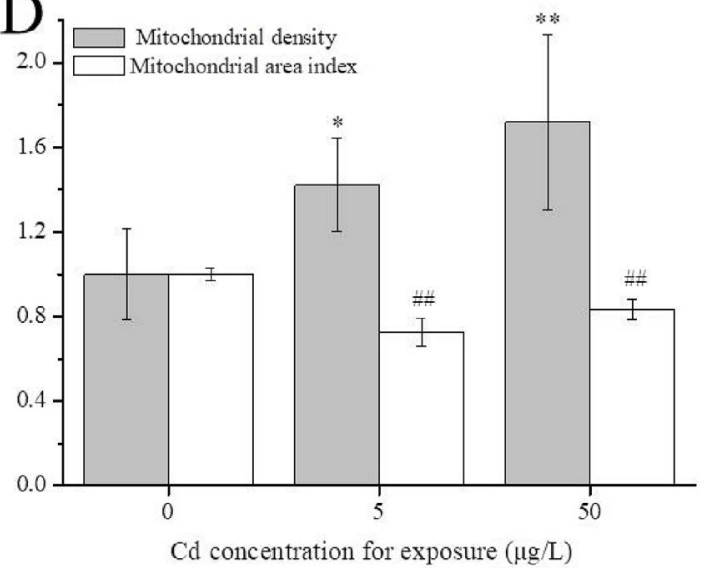

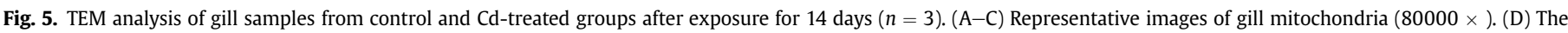

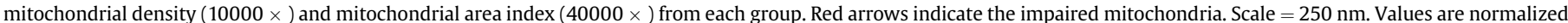

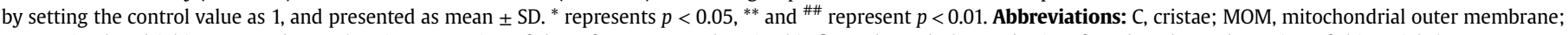
MIM, mitochondrial inner membrane. (For interpretation of the references to colour in this figure legend, the reader is referred to the Web version of this article.)

Cd-treated groups, including mitochondrial intermembrane space import and assembly protein 40 (MIA40), sorting and assembly machinery component 50 homolog (SAMM50), YidC/Oxa1 family membrane protein insertase (Oxa1), three mitochondrial import inner membrane translocase subunits (Tim9, Tim17A and Tim21), and two metaxin proteins (MTX1 and MTX2). Tim9, Tim17A, Tim21, Oxa1, MTX1 and MTX2, are involved in protein transportation into mitochondria (Armstrong et al., 1999; Meier et al., 2005; Muhlenbein et al., 2004; Sato and Mihara, 2009). MIA40 is required for the translocation and folding of small cysteine-containing proteins (small Tim), and Sam50 is crucial for maintaining the structure of cristae and the proper assembly of respiratory chain complexes (Banci et al., 2009; Ott et al., 2012). In this work, Tim9, Tim17A, MIA40, and MTX2 were commonly up-regulated in both Cd-treated groups. Oxa1, SAMM50, and MTX1 were uniquely upregulated in the low concentration $(5 \mu \mathrm{g} / \mathrm{L})$ of Cd-treated group, while Tim 21 was up-regulated in the high concentration $(50 \mu \mathrm{g} / \mathrm{L})$ of Cd-treated group only. These DEPs indicated that mitochondrial import was altered to adapt $\mathrm{Cd}$ exposures.

Many interacting proteins of MICOS complex have been identified in mitochondria of yeast and mammalian. MIC60 interacts with MTX1, MTX2, SAM50, MIA40, MIC19, MIC25 and DnaJC11, demonstrating the vital role of MIC60 in mitochondrial protein import and transport (Xie et al., 2007; Zerbes et al., 2012). MIC19 bridges MIC60 and SAM50, contributing to the formation of contact sites (Quintana-Cabrera et al., 2018). It was reported that MIC19 might regulate protein transport through SAM complex and regulate protein processing by binding to MIA40 (Darshi et al., 2011). In this work, impaired cristae and mitochondrial networks were observed in flounders from both Cd treatments. Therefore, the upregulation of these proteins related to mitochondrial morphology and import might act as a compensation mechanism to adapt $\mathrm{Cd}$ exposure in flounder gills.

\subsection{Effects of $\mathrm{Cd}$ on energy metabolism}

\subsubsection{TCA cycle}

TCA cycle is the ultimate metabolic pathway and the metabolic hub of carbohydrates, lipids and amino acids in aerobic organisms. In this work, several DEPs related to TCA cycle were identified in both Cd-treated groups (Fig. 6). NAD (P) transhydrogenase (NNT) catalyzes the transhydrogenation from $\mathrm{NAD}^{+}$to NADPH, and coordinates the contribution of glutamine (Gln) to TCA cycle and glucose catabolism (Gameiro, 2013). It was up-regulated in both Cd-treated groups. Consistently, six DEPs involved in Gln metabolism and glucose catabolism were altered by $\mathrm{Cd}$ treatments. Hexokinase-1 (HK1) is an allosteric enzyme phosphorylating glucose to glucose-6-phosphate (Coerver et al., 1997). Mitochondrial pyruvate carrier (Mpc1 and $\mathrm{Mpc} 2$ ) and mitochondrial 2oxoglutarate/malate carrier protein (SLC25A11) were associated with the transport of pyruvate and malate into mitochondria respectively (Gallo et al., 2011; Sébastien et al., 2012). Mpc1, Mpc2 


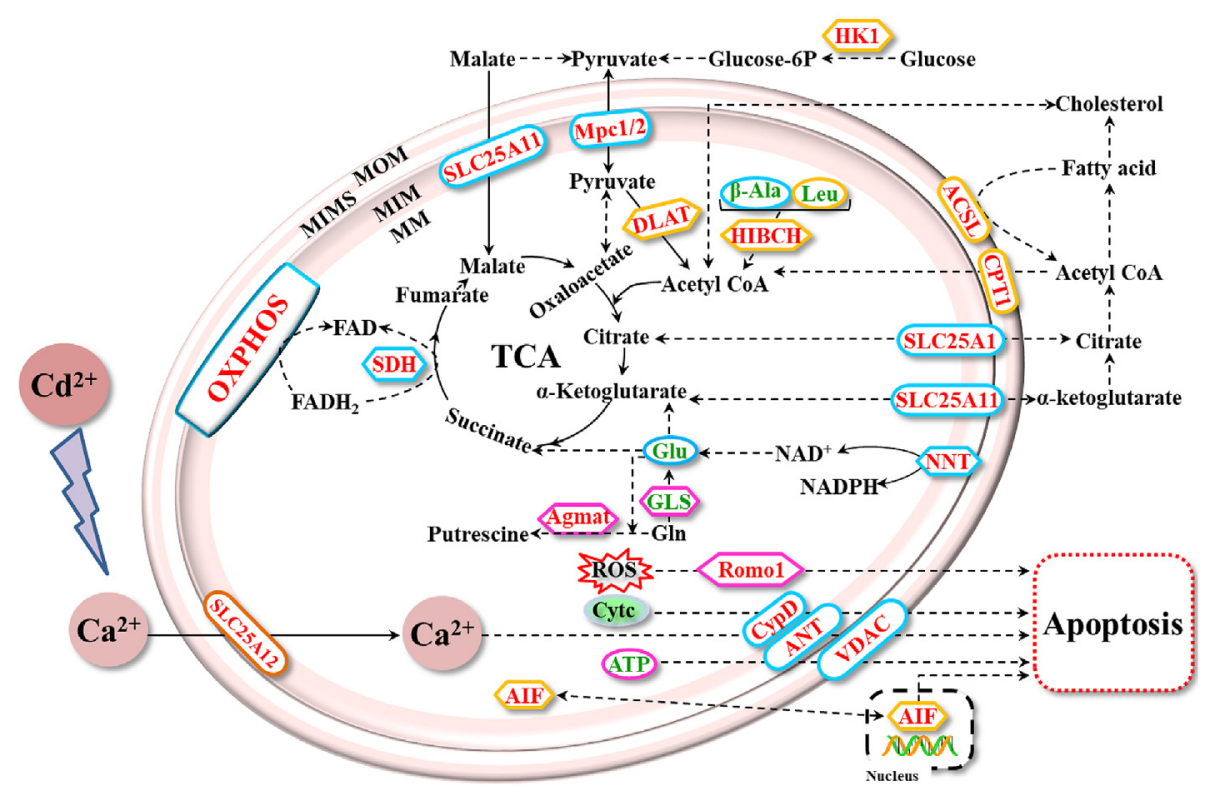

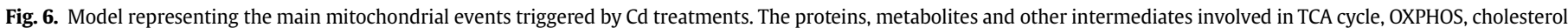

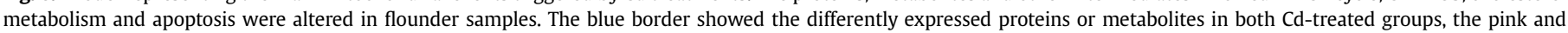

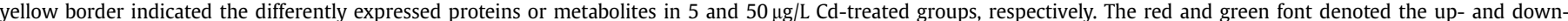

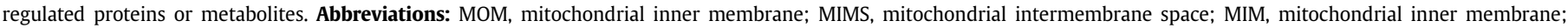

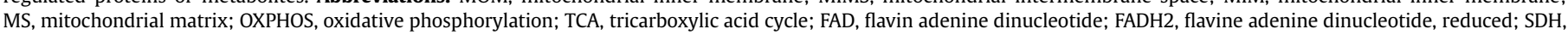

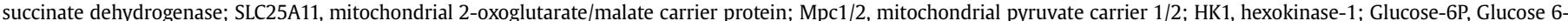

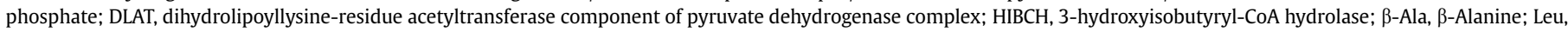

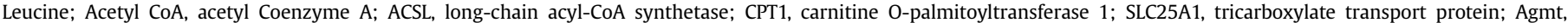

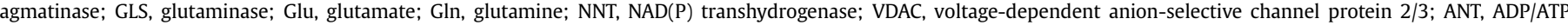

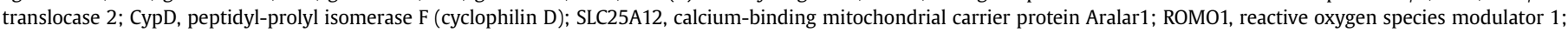
AIF, apoptosis-inducing factor 1. (For interpretation of the references to colour in this figure legend, the reader is referred to the Web version of this article.)

and SLC25A11 were significantly up-regulated in both Cd treatments and HK1 was up-regulated in the high concentration $(50 \mu \mathrm{g} /$ L) of Cd-treated group, indicating the promoted glycolysis by $\mathrm{Cd}$ exposure. Glutaminase (GLS) could promote mitochondrial respiration and increase ATP generation by catalyzing the synthesis of glutamate (Glu) and $\alpha$-ketoglutarate (Hu et al., 2010). Agmatinase (Agmat) was responsible for conversion of Gln to putrescine (Iyer et al., 2002). GLS was down-regulated and Agmat was upregulated in the low concentration $(5 \mu \mathrm{g} / \mathrm{L})$ of $\mathrm{Cd}$ treatment, while metabolomic profile exhibited decreased Glu level in both $\mathrm{Cd}$ treated groups (Fig. S2). In some cases, Gln and glucose were reported to maintain TCA cycle in complementary fashion (Yang et al., 2014). These proteins and metabolites, including HK1, Mpc1, Mpc2, SLC25A11, GLS, Agmat, and Glu, demonstrated that the contribution of Gln to the TCA cycle was inhibited and the glucose metabolism was enhanced in gill mitochondria of flounders exposed to $\mathrm{Cd}$. These findings were similarly observed in mitochondria of clam Ruditapes philippinarum treated by Cd (Ji et al., 2019). Long-chain acyl-CoA synthetase (ACSL) and carnitine O-palmitoyltransferase $1 \mathrm{~A}$ (CPT1A), involved in $\beta$-fatty acid oxidation, are associated with the production of acetyl-CoA (Bennett et al., 2004; Mashek et al., 2007). Dihydrolipoyllysine-residue acetyltransferase component of pyruvate dehydrogenase complex (DLAT) and 3hydroxyisobutyryl-CoA hydrolase ( $\mathrm{HIBCH})$ catalyze the conversion of pyruvate or amino acids to acetyl-CoA (Hawes et al., 1996; Head et al., 2010). These proteins, including DLAT, HIBCH, ACSL and CPT1A, were uniquely up-regulated in the high concentration $(50 \mu \mathrm{g} / \mathrm{L})$ of $\mathrm{Cd}$-treated group, suggesting an obvious promotion of TCA cycle compared with the low concentration $(5 \mu \mathrm{g} / \mathrm{L})$ of $\mathrm{Cd}$ treated group. The significantly altered proteins and metabolites related to TCA cycle implied the increased demand of ATP for Cd resistance (Fig. 6).

\subsubsection{Oxidative phosphorylation}

Oxidative phosphorylation (OXPHOS), occurring in the MIM of eukaryotic cells, provides most of the energy for cellular functions in a highly efficient way (Senior, 1988). OXPHOS is mainly composed of five complexes, including complex I, succinate dehydrogenase (complex II), complex III, complex IV, and ATP synthase (complex V). Five OXPHOS complexes were significantly upregulated in both Cd-treated groups. Complex I and Complex II played vital roles in the mitochondrial electron transport chain (ETC) because they are the initial entry sites of reducing energy (via NADH or $\mathrm{FADH}_{2}$ ) into OXPHOS (Hirst, 2013; Horsefield et al., 2004). In total, 32 subunits of complex I and all subunits of complex II were up-regulated in both Cd treatments (Table S1). Noteworthily, DEPs with a larger number and higher fold changes were induced by the high concentration $(50 \mu \mathrm{g} / \mathrm{L})$ of $\mathrm{Cd}$, indicating more severe impacts on enhancing electron transfer and contribution of molecules into OXPHOS induced by the high concentration $(50 \mu \mathrm{g} / \mathrm{L})$ of Cd. Moreover, complex II is the only enzyme that connects the TCA cycle with the respiratory chain (Horsefield et al., 2006). The upregulated complex II was related to the up-regulated TCA cycle induced by Cd. Complex II and complex III are the main sites for ROS generation in ETC (Guzy et al., 2005; Renate et al., 2003). Five and seven subunits of complex III were respectively up-regulated in both Cd treatments (Table S1), implying the overproduction of ROS. Complex IV is responsible for the electron transfer from reduced cytochrome $c$ to molecular oxygen. Six and seven subunits of complex IV were respectively up-regulated in the low and high concentration of Cd-treated groups (Table S1), suggesting the increased oxygen consumption. Complex $\mathrm{V}$ is the final enzyme in 
the OXPHOS, which uses proton gradients across a membrane to synthesize ATP from ADP (Daichi et al., 2011). In complex V, 9 and 13 subunits were respectively up-regulated in the low and high concentrations of Cd treatments (Table S1), which meant the increased ATP production caused by $\mathrm{Cd}$ in flounder gills. However, metabolomic analysis revealed the decreased ATP level in both Cdtreated samples (Fig. S2). Complex V was also reported to scaffold the mitochondrial cristae (Johann et al., 2013). MICOS components can cluster with complex V and they coordinate to bend the MIM and maintain the mitochondrial ultrastructure (Jimenez et al., 2014). Thus, the up-regulated complex V should be involved in maintaining mitochondrial morphology rather than promoting ATP production. And the energy depletion might be attributed to substrate mobilization and mitochondrial impairment. Similarly, this phenomenon was also observed in hepatopancreas of freshwater crabs (Sinopotamon henanense) induced by Cd (Yang et al., 2015). Taken together, an enhancement of the electron flux within gill mitochondria was induced by $\mathrm{Cd}$ treatments, implying the enhanced energy demands in flounder gills.

\subsection{Effects of $\mathrm{Cd}$ on primary bile acid biosynthesis}

Excess cholesterol is mainly converted into primary bile acids (BAs) and secondary BAs in liver and intestine and eliminated from the body subsequently, in which cytochrome P450s (CYPs) play crucial roles (Lorbek et al., 2012). In general, the primary BAs (cholate and chenodeoxycholate) could be produced by the neutral pathway, the acidic pathway, and 24- or 25-hydroxycholesterol ( $240 \mathrm{OH}$ or $25 \mathrm{OH}$ ) pathway (Baila-Rueda et al., 2014). Interestingly, cholate was increased in gill samples after the low concentration $(5 \mu \mathrm{g} / \mathrm{L})$ of Cd exposure according to metabolomic profile (Fig. S2B). It is worth noting that cholestanetriol 26-monooxygenase (CYP27A1) in the acidic pathway is ubiquitously expressed in mitochondria (Pikuleva et al., 1998). CYP27A1, involved in the primary BA biosynthesis pathway, was down-regulated in $5 \mu \mathrm{g} / \mathrm{L} \mathrm{Cd}-$ treated group (Table S1). Researches revealed that BAs of endogenous hydrophobic or from ectogenous dietetic sources suppressed BA synthesis and the activities and mRNA levels of CYPs, such as CYP27A1 (Li-Hawkins et al., 2002). Twisk et al. (2010) suggested that the inhibitory potency was related to the position of hydroxyl groups in the three-dimensional structure of BAs. Therefore, the increased cholate level and down-regulated primary bile acid biosynthesis pathway might be related to the negative feedback on primary BA biosynthesis of cholate in flounder gills treated with the low concentration $(5 \mu \mathrm{g} / \mathrm{L})$ of $\mathrm{Cd}$. As same as cholate, myo-inositol is believed to play a vital role in cholesterol homeostasis (Kotaki et al., 1968). Increased myo-inositol level was observed in the metabolomic profile of flounder samples from the high concentration $(50 \mu \mathrm{g} / \mathrm{L})$ of Cd-treated group. It was reported that BAs could induce mitochondrial toxicity (Palmeira and Rolo, 2004). Thus, the upregulation of myo-inositol induced by the high concentration $(50 \mu \mathrm{g} / \mathrm{L})$ of $\mathrm{Cd}$ might not only be a different metabolic pathway to prevent the excessive storage of cholesterol, but also a strategy for reducing mitochondrial damage induced by cholate.

\subsection{Effects of $\mathrm{Cd}$ on stress resistance and apoptosis}

Apart from generating energy, mitochondria also play a crucial role in resisting to environmental stress. In both Cd-treated groups, several DEPs related to stress resistance and apoptosis were significantly altered (Fig. 6). Reactive oxygen species modulator 1 (Romo1) could induce ROS production in mitochondria (Chung et al., 2006). The up-regulation of Romo1 in the low concentration $(5 \mu \mathrm{g} / \mathrm{L})$ of Cd-treated group suggested more ROS production, which was consistent with the up-regulated complex II and III. The overproduction of ROS could not only suppress the ATP generation via acting on MMP and ETC, but also overload $\mathrm{Ca}^{2+}$ level in cells (Biswas et al., 1999). Interestingly, six relevant DEPs were identified in Cd-treated samples. Mitochondrial carrier protein 1 (UCP5), a mitochondrial uncoupling carrier, participates in the mitochondrial proton leak (Kondou et al., 2000). UCP5 was up-regulated in the high concentration $(50 \mu \mathrm{g} / \mathrm{L})$ of Cd-treated group, which meant reduced MMP and ATP production as indicated by MMP detection (Fig. 3) and metabolomic profile (Fig. S2D). Protein NipSnap homolog 2 (NIPSNAP2) may act as a positive regulator of L-type calcium channels (Wang et al., 1998). The up-regulation of NIPSNAP2 possibly contributed to the overload of intracellular $\mathrm{Ca}^{2+}$ induced by high concentration $(50 \mu \mathrm{g} / \mathrm{L})$ of $\mathrm{Cd}$ in flounder gills. The opening of mitochondrial permeability transition pore (MPTP) led to a decrease in MMP and the outflow of $\mathrm{Ca}^{2+}$ and cytochrome $c$ from mitochondria (Naranmandura et al., 2012). Voltage-dependent anion-selective channel protein (VDAC2 and VDAC3), peptidylprolyl isomerase $\mathrm{F}(\mathrm{CypD})$, and $\mathrm{ADP} / \mathrm{ATP}$ translocase 2 (ANT) were believed to be the components of MPTP (Rasola and Bernardi, 2007). Four up-regulated proteins, VDAC2, VDAC3, CypD and ANT, in both $\mathrm{Cd}$ treatments implied more influxes and effluxes through mPTP, which might enhance the mitochondrial membrane permeability and decrease the MMP. Once beyond the regulatory capacity of mitochondria, apoptosis would be triggered by external environmental stress (Wu et al., 2014). In total, three DEPs related to apoptosis, bcl2-associated agonist of cell death (BAD), induced myeloid leukemia cell differentiation protein Mcl-1 homolog (Mcl1), and apoptosis-inducing factor 1 (AIF), were altered in Cdtreated groups. $\mathrm{Bcl} 2$ family is the central regulator of caspase activation, and its opposing factions of anti- and pro-apoptotic members arbitrate the life-or-death decision (Suzanne and Adams, 2002). Mcl1 is an anti-apoptotic protein, while BAD is a pro-apoptotic protein. These two proteins could regulate the mitochondrial membrane permeability and the opening of the mPTP (Hollville et al., 2014; Maurer et al., 2006). The up-regulation of BAD and down-regulation of Mcl1 in the low concentration $(5 \mu \mathrm{g} / \mathrm{L})$ of $\mathrm{Cd}$-treated group would promote the mitochondrial depolarization and the opening of MPTP. Once MPTP was opened, cytochrome $c$ and AIF would release from mitochondria, initiating the apoptosis (Son et al., 2009). Interestingly, AIF was up-regulated in the high concentration $(50 \mu \mathrm{g} / \mathrm{L})$ of $\mathrm{Cd}$ treatment. The changes of these proteins suggested that the low and high concentrations of $\mathrm{Cd}$ might impact on stress resistance and apoptosis in juvenile flounders via differential pathways.

\section{Conclusion}

In this work, the global responses in mitochondrial morphology and mitochondrial import, TCA cycle, OXPHOS, primary bile acid biosynthesis, stress resistance, and apoptosis were characterized in gill mitochondria from juvenile flounder $P$. olivaceus exposed to $\mathrm{Cd}$ for 14 days. Moreover, more and smaller mitochondria and significantly decreased MMPs were observed in Cd-treated flounder samples. These results indicated that mitochondria were the key target of Cd toxicity, and dynamic regulations of energy homeostasis, cholesterol metabolism, stress resistance, apoptosis, and mitochondrial morphology in gill mitochondria might play significant roles in coping strategy of flounders to $\mathrm{Cd}$. Overall, this work demonstrated a comprehensive characterization on mitochondrial toxicity of $\mathrm{Cd}$ in flounder gills, providing new insights into the mechanism of Cd-induced toxicity in marine fishes.

\section{Ethical statement}

All procedures were strictly performed according to the 
Guidelines of the Chinese Council on Laboratory Animal Care (2011), which was approved by the Animal Research Ethics Board of Chinese Academy of Sciences.

\section{Declaration of competing interest}

We declare that we do not have any commercial or associative interest that represents a conflict of interest in connection with the work submitted.

\section{Acknowledgments}

This work was supported by NSFC (41676114), the Young Taishan Scholars Program of Shandong Province for Prof. Huifeng Wu (tsqn201812115) and Qingdao National Laboratory for Marine Science and Technology (QNLM201701). We thank Prof. Mark Viant for the use of ProMetab software.

\section{Appendix A. Supplementary data}

Supplementary data to this article can be found online at https://doi.org/10.1016/j.envpol.2019.113591.

\section{References}

An, J., Shi, J., He, Q., et al., 2012. CHCM1/CHCHD6, novel mitochondrial protein linked to regulation of mitofilin and mitochondrial cristae morphology. J. Biol. Chem. 287 (10), 7411-7426.

Armstrong, L.C., Saenz, A.J., Bornstein, P., 1999. Metaxin 1 interacts with metaxin 2, a novel related protein associated with the mammalian mitochondrial outer membrane. J. Cell. Biochem. 74 (1), 11-22.

Bai, S., Xu, Z., 2006. Effects of cadmium on mitochondrion structure and energy metabolism of Pelteobagrus fulvidraco gill (in Chinese). Chin. J. Appl. Ecol. 17 (7), 1213-1217.

Baila-Rueda, L., Mateo-Gallego, R., Jarauta, E., et al., 2014. Bile acid synthesis precursors in familial combined hyperlipidemia: the oxysterols 24S-hydroxycholestero1 and 27-hydroxycholesterol. Biochem. Biophys. Res. Commun. 446 (3), 731-735.

Banci, L., Bertini, I., Cefaro, C., et al., 2009. MIA40 is an oxidoreductase that catalyzes oxidative protein folding in mitochondria. Nat. Struct. Mol. Biol. 16 (2), 198-206.

Bannwarth, S., Ait-El-Mkadem, S., Chaussenot, A., et al., 2014. A mitochondrial origin for frontotemporal dementia and amyotrophic lateral sclerosis through CHCHD10 involvement. Brain 137 (8), 2329-2345.

Bennett, M.J., Boriack, R.L., Narayan, S., et al., 2004. Novel mutations in CPT 1A define molecular heterogeneity of hepatic carnitine palmitoyltransferase I deficiency. Mol. Genet. Metab. 82 (1), 59-63.

Biswas, G., Adebanjo, O.A., Freedman, B.D., et al., 1999. Retrograde $\mathrm{Ca}^{2+}$ signaling in C2C12 skeletal myocytes in response to mitochondrial genetic and metabolic stress: a novel mode of inter-organelle crosstalk. EMBO J. 18 (3), 522-533.

Cao, L., Huang, W., Shan, X., et al., 2012. Tissue-specific accumulation of cadmium and its effects on antioxidative responses in Japanese flounder juveniles. Environ. Toxicol. Pharmacol. 33 (1), 16-25.

Chandurvelan, R., Marsden, I.D., Gaw, S., et al., 2017. Acute and sub-chronic effects of sub-lethal cadmium exposure on energy metabolism in the freshwater shrimp Paratya curvirostris. Ecotoxicol. Environ. Saf. 135, 60-67.

Chung, Y.M., Kim, J.S., Yoo, Y.D., 2006. A novel protein, Romo1, induces ROS production in the mitochondria. Biochem. Biophys. Res. Commun. 347 (3) 649-655.

Coerver, K.A., Gray, S.M., Barnes, J.E., et al., 1997. Developmental expression of hexokinase 1 and 3 in rats. Histochem. Cell Biol. 109 (1), 75-86.

Cui, L., Wang, S., Gao, L., et al., 2018. Concentrations and trophic magnification of polychlorinated naphthalenes (PCNs) in marine fish from the Bohai coasta area, China. Environ. Pollut. 234, 876-884.

Daichi, O., Ryota, I., Hiroyuki, N., 2011. Rotation and structure of $\mathrm{F}_{0} \mathrm{~F}_{1}$-ATP synthase. J. Biochem. 149 (6), 655-664.

Darshi, M., Mendiola, V.L., Mackey, M.R, et al, 2011. ChChd3, an inner mitochondrial membrane protein, is essential for maintaining crista integrity and mitochondrial function. J. Biol. Chem. 286 (4), 2918-2932.

Ding, C., Wu, Z., Huang, L., et al., 2015. Mitofilin and CHCHD6 physically interact with Sam50 to sustain cristae structure. Sci. Rep. 5 (1), 16064.

Dorta, D.J., Leite, S., Demarco, K.C., et al., 2003. A proposed sequence of events for cadmium-induced mitochondrial impairment. J. Inorg. Biochem. 97 (3) $251-257$.

El-Ebiary, E., Wahbi, O., El-Greisy, Z., 2013. Influence of dietary Cadmium on sexual maturity and reproduction of Red Tilapia. The Egypt. J. Aquat. Res. 39 (4), 313-317.
Friedman, J.R., Mourier, A., Yamada, J., et al., 2015. MICOS coordinates with respiratory complexes and lipids to establish mitochondrial inner membrane architecture, eLife 4, e07739.

Gallo, M., Park, D., Luciani, D.S., et al., 2011. MISC-1/OGC links mitochondrial metabolism, apoptosis and insulin secretion. PLoS One 6 (3), e17827.

Gameiro, P.A., 2013. Cofactor balance by nicotinamide nucleotide transhydrogenase (NNT) coordinates reductive carboxylation and glucose catabolism in the tricarboxylic acid (TCA) cycle. J. Biol. Chem. 288 (18), 12967-12977.

Gao, X., Zhou, F., Chen, C.-T.A., 2014. Pollution status of the Bohai Sea: an overview of the environmental quality assessment related trace metals. Environ. Int. 62. $12-30$.

Genin, E.C., Plutino, M., Bannwarth, S., et al., 2016. CHCHD10 mutations promote loss of mitochondrial cristae junctions with impaired mitochondrial genome maintenance and inhibition of apoptosis. EMBO Mol. Med. 8 (1), 58-72.

Glytsou, C., Calvo, E., Cogliati, S., et al., 2016. Optic atrophy 1 is epistatic to the core MICOS component MIC60 in mitochondrial cristae shape control. Cell Rep. 17 (11), 3024-3034.

Guzy, R.D., Hoyos, B., Robin, E., et al., 2005. Mitochondrial complex III is required for hypoxia-induced ROS production and cellular oxygen sensing. Cell Metabol. 1 (6), 401-408.

Harner, M., Körner, C., Walther, D., et al., 2011. The mitochondrial contact site complex, a determinant of mitochondrial architecture. EMBO J. 30 (21), 4356-4370.

Hawes, J.W., Jaskiewicz, J., Shimomura, Y., et al., 1996. Primary structure and tissuespecific expression of human beta-hydroxyisobutyryl-coenzyme A hydrolase. J. Biol. Chem. 271 (42), 26430-26434.

Head, R.A., Brown, R.M., Zarazuela, Z., et al., 2010. Clinical and genetic spectrum of pyruvate dehydrogenase deficiency: dihydrolipoamide acetyltransferase (E2) deficiency. Ann. Neurol. 58 (2), 234-241.

Hirst, J., 2013. Mitochondrial complex I. Annu. Rev. Biochem. 82 (1), 551-575.

Hollville, E., Carroll, R., Cullen, S., et al., 2014. Bcl-2 family proteins participate in mitochondrial quality control by regulating Parkin/PINK1-dependent mitophagy. Mol. Cell 55 (3), 451-466.

Horsefield, R. Iwata, S., Byrne, B., 2004. Complex II from a structural perspective. Curr. Protein Pept. Sci. 5 (2), 107-118.

Horsefield, R., Yankovskaya, V., Sexton, G., et al., 2006. Structural and computational analysis of the quinone-binding site of complex II (succinate-ubiquinone oxidoreductase): a mechanism of electron transfer and proton conduction during ubiquinone reduction. J. Biol. Chem. 281 (11), 7309-7316.

Hu, W., Zhang, C., Wu, R., et al., 2010. Glutaminase 2, a novel p53 target gene regulating energy metabolism and antioxidant function. Proc. Natl. Acad. Sci. U. S. A 107 (16), 7455-7460.

Iyer, R.K., Kim, H.K., Tsoa, R.W., et al., 2002. Cloning and characterization of human agmatinase. Mol. Genet. Metab. 75 (3), 209-218.

Ji, C., Lu, Z., Xu, L., et al., 2019. Evaluation of mitochondrial toxicity of cadmium in clam Ruditapes philippinarum using iTRAQ-based proteomics. Environ. Pollut. 251, 802-810.

Ji, C., Wu, H., Wei, L., et al., 2013. Proteomic and metabolomic analysis of earthworm Eisenia fetida exposed to different concentrations of 2,2',4,4'-tetrabromodiphenyl ether. J. Proteom. 91, 405-416.

Jimenez, L., Laporte, D., Duvezin-Caubet, S., et al., 2014. Mitochondrial ATP synthases cluster as discrete domains that reorganize with the cellular demand for oxidative phosphorylation. J. Cell Sci. 127 (4), 719-726.

Jin, Y., Liu, Z., Liu, F., et al., 2015. Embryonic exposure to cadmium (II) and chromium (VI) induce behavioral alterations, oxidative stress and immunotoxicity in zebrafish (Danio rerio). Neurotoxicol. Teratol. 48, 9-17.

Johann, H., Isabelle, L., Muriel, P., et al., 2013. Human $\mathrm{F}_{1} \mathrm{~F}_{0}$ ATP synthase, mitochondrial ultrastructure and OXPHOS Impairment: a (Super-)Complex matter? PLoS One 8 (10), e75429.

Jurss, K., Bastrop, R., 1995. The function of mitochondria-rich cells (chloride cells) in teleost gills. Rev. Fish Biol. Fish. 5 (2), 235-255.

Karbowski, M., Youle, R.J., 2003. Dynamics of mitochondrial morphology in healthy cells and during apoptosis. Cell Death Differ. 10 (8), 870-880.

Kim, S.-G., Jee, J.-H., Kang, J.-C., 2004. Cadmium accumulation and elimination in tissues of juvenile olive flounder, Paralichthys olivaceus after sub-chronic cadmium exposure. Environ. Pollut. 127 (1), 117-123.

Kondou, S., Hidaka, S., Yoshimatsu, H., et al., 2000. Molecular cloning of rat brain mitochondrial carrier protein-1 cDNA and its up-regulation during postnatal development. Biochim. Biophys. Acta Bioenerg. 1457 (3), 182-189.

Kotaki, A., Sakurai, T., Kobayashi, M., et al., 1968. Studies on myoinositol. IV. Effect of myoinositol on cholesterol metabolism of rats suffering from experimental fatty liver. J. Vitaminol. 14 (2), 87-94.

Lane, T.W., Morel, F.M.M., 2000. A biological function for cadmium in marine diatoms. Proc. Natl. Acad. Sci. 97, 4627-4631.

Leahy, J., Spahis, S., Bonneil, E., et al., 2018. Insight from mitochondrial functions and proteomics to understand cardiometabolic disorders in survivors of acute lymphoblastic leukemia. Metab. Clin. Exp. 85, 151-160.

Li, H., Gao, X., Gu, Y., et al., 2018. Comprehensive large-scale investigation and assessment of trace metal in the coastal sediments of Bohai Sea. Mar. Pollut. Bull. 129 (1), 126-134

Li-Hawkins, J., Gafvels, M., Olin, M., et al., 2002. Cholic acid mediates negative feedback regulation of bile acid synthesis in mice. J. Clin. Investig. 110 (8), 1191-1200.

Ling, X.P., Zhu, J.Y., Huang, L., et al., 2009. Proteomic changes in response to acute cadmium toxicity in gill tissue of Paralichthys olivaceus. Environ. Toxicol. 
Pharmacol. 27 (2), 212-218.

Lorbek, G., Lewinska, M., Rozman, D., 2012. Cytochrome P450s in the synthesis of cholesterol and bile acids. FEBS J. 279 (9), 1516-1533.

Mashek, D.G., Li, L.O., Coleman, R.A., 2007. Long-chain acyl-CoA synthetases and fatty acid channeling. Future Lipidol. 2 (4), 465-476.

Maurer, U., Charvet, C., Wagman, A.S., et al., 2006. Glycogen synthase kinase-3 regulates mitochondrial outer membrane permeabilization and apoptosis by destabilization of MCL-1. Mol. Cell 21 (6), 749-760.

Meier, S., Neupert, W., Herrmann, J.M., 2005. Conserved N-terminal negative charges in the Tim17 subunit of the TIM23 translocase play a critical role in the import of preproteins into mitochondria. J. Biol. Chem. 280 (9), 7777-7785.

Meyer, J.N., Leung, M.C.K., Rooney, J.P., et al., 2013. Mitochondria as a target of environmental toxicants. Toxicol. Sci. 134 (1), 1-17.

Muhlenbein, N., Hofmann, S., Rothbauer, U., et al., 2004. Organization and function of the small Tim complexes acting along the import pathway of metabolite carriers into mammalian mitochondria. J. Biol. Chem. 279 (14), 13540-13546.

Naranmandura, H., Chen, X., Tanaka, M., et al., 2012. Release of apoptotic cytochrome $c$ from mitochondria by dimethylarsinous acid occurs through interaction with voltage-dependent anion channel in Vitro. Toxicol. Sci. 128 (1), 137-146.

Ott, C., Ross, K., Straub, S., et al., 2012. Sam50 functions in mitochondrial intermembrane space bridging and biogenesis of respiratory complexes. Mol. Cell. Biol. 32 (6), 1173-1188.

Palmeira, C.M., Rolo, A.P., 2004. Mitochondrially-mediated toxicity of bile acids. Toxicology 203 (1), 1-15.

Perelman, A., Wachtel, C., Cohen, M., et al., 2012. JC-1: alternative excitation wavelengths facilitate mitochondrial membrane potential cytometry. Cell Death Dis. 3 (11), e430.

Pikuleva, I.A., Babiker, A., Waterman, M.R., et al., 1998. Activities of recombinant human cytochrome P450c27 (CYP27) which produce intermediates of alternative bile acid biosynthetic pathways. J. Biol. Chem. 273 (29), 18153-18160.

Quintana-Cabrera, R., Mehrotra, A., Rigoni, G., et al., 2018. Who and how in the regulation of mitochondrial cristae shape and function. Biochem. Biophys. Res. Commun. 500 (1), 94-101.

Rasola, A., Bernardi, P., 2007. The mitochondrial permeability transition pore and its involvement in cell death and in disease pathogenesis. Apoptosis 12 (5), 815-833.

Renate, P., Barat, I., Anna, G., et al., 2003. Essential role of complex II of the respiratory chain in hypoxia-induced ROS generation in the pulmonary vasculature. Am. J. Physiol. Lung Cell Mol. Physiol. 284 (5), L710-L719.

Sanadi, D.R., Hughes, J.B., Joshi, S., 1981. Activation of potassium-dependent $\mathrm{H}^{+}$ efflux from mitochondria by cadmium and phenylarsine oxide. J. Bioenerg. Biomembr. 13 (5-6), 425-431.

Sato, T., Mihara, K., 2009. Topogenesis of mammalian Oxa1, a component of the mitochondrial inner membrane protein export machinery. J. Biol. Chem. 284 (22), 14819-14827.

Sébastien, H., Etienne, R., Sylvie, M., et al., 2012. Identification and functional expression of the mitochondrial pyruvate carrier. Science 337 (6090), 93-96.

Senior, A.E., 1988. ATP synthesis by oxidative phosphorylation. Physiol. Rev. 68 (1), $177-231$.

Sokolova, I.M., Sokolov, E.P., Ponnappa, K.M., 2005a. Cadmium exposure affects mitochondrial bioenergetics and gene expression of key mitochondrial proteins in the eastern oyster Crassostrea virginica Gmelin (Bivalvia: Ostreidae). Aquat.
Toxicol. 73 (3), 242-255.

Sokolova, I.M., Ringwood, A.H., Johnson, C., 2005b. Tissue-specific accumulation of cadmium in subcellular compartments of eastern oysters Crassostrea virginica Gmelin (Bivalvia: Ostreidae). Aquat. Toxicol. 74 (3), 218-228.

Son, Y., Jang, Y., Heo, J., et al., 2009. Apoptosis-inducing factor plays a critical role in caspase-independent, pyknotic cell death in hydrogen peroxide-exposed cells. Apoptosis 14 (6), 796-808.

Suzanne, C., Adams, J.M., 2002. The Bcl2 family: regulators of the cellular life-ordeath switch. Nat. Rev. Cancer 2 (9), 647-656.

Thophon, S., Pokethitiyook, P., Chalermwat, K., et al., 2010. Ultrastructural alterations in the liver and kidney of white sea bass, Lates calcarifer, in acute and subchronic cadmium exposure. Environ. Toxicol. Chem. 19 (1), 11-19.

Trevisan, R., Delapedra, G., Mello, D.F., et al., 2014. Gills are an initial target of zinc oxide nanoparticles in oysters Crassostrea gigas, leading to mitochondrial disruption and oxidative stress. Aquat. Toxicol. 153, 27-38.

Twisk, J., Hoekman, M.F., Muller, L.M., et al., 2010. Structural aspects of bile acids involved in the regulation of cholesterol 7 alpha-hydroxylase and sterol 27 hydroxylase. FEBS J. 228 (3), 596-604.

Wang, X.Y., Smith, D.I., Liu, W., et al., 1998. GBAS, a novel gene encoding a protein with tyrosine phosphorylation sites and a transmembrane domain, is coamplified with EGFR. Genomics 49 (3), 448-451.

Wu, H., Che, X., Zheng, Q., et al., 2014. Caspases: a molecular switch node in the crosstalk between autophagy and apoptosis. Int. J. Biol. Sci. 10 (9), 1072-1083.

Wu, H., Wang, W., 2010. NMR-based metabolomic studies on the toxicological effects of cadmium and copper on green mussels Perna viridis. Aquat. Toxicol. 100 (4), 339-345.

Xia, L., Chen, S., Dahms, H.U., et al., 2016. Cadmium induced oxidative damage and apoptosis in the hepatopancreas of Meretrix meretrix. Ecotoxicology 25 (5), 959-969.

Xie, J., Marusich, M.F., Souda, P., et al., 2007. The mitochondrial inner membrane protein Mitofilin exists as a complex with SAM50, metaxins 1 and 2, coiled-coilhelix coiled-coil-helix domain-containing protein 3 and 6 and DnaJC11. FEBS (Fed. Eur. Biochem. Soc.) Lett. 581 (18), 3545-3549.

Xuan, R., Wu, H., Li, Y., et al., 2014. Sublethal Cd-induced cellular damage and metabolic changes in the freshwater crab Sinopotamon henanense. Environ. Sci. Pollut. Res. Int. 21 (3), 1738-1745.

Yang, C., Ko, B., Hensley, C.T., et al., 2014. Glutamine oxidation maintains the TCA cycle and cell survival during impaired mitochondrial pyruvate transport. Mol. Cell 56 (3), 414-424.

Yang, J., Liu, D., He, Y., et al., 2015. Mitochondrial energy metabolism in the hepatopancreas of freshwater crabs (Sinopotamon henanense) after cadmium exposure. Environ. Sci.: Process. Impacts 17 (1), 156-165.

Zarouchlioti, C., Parfitt, D.A., Li, W., et al., 2018. DNAJ Proteins in neurodegeneration: essential and protective factors. Philos. Trans. R. Soc. Biol. Sci. 372 (1738), 20160534.

Zerbes, R.M., Bohnert, M., Stroud, D.A., et al., 2012. Role of MINOS in mitochondrial membrane architecture: cristae morphology and outer membrane interactions differentially depend on mitofilin domains. J. Mol. Biol. 422 (2), 183-191.

Zhu, J.Y., Huang, H.Q., Bao, X.D., et al., 2006. Acute toxicity profile of cadmium revealed by proteomics in brain tissue of Paralichthys olivaceus: potential role of transferrin in cadmium toxicity. Aquat. Toxicol. 78 (2), 127-135.

Zorova, L.D., Popkov, V.A., Plotnikov, E.Y., et al., 2018. Mitochondrial membrane potential. Anal. Biochem. 552, 50-59. 